\title{
Current state and potential of Balloon Sinuplasty
}

\author{
Raymond L. Weiss
}

Sinuplasty Center of Excellence, Biloxi, MS, USA

Functional Endoscopic Sinus Surgery (FESS) has become the accepted option for treating medically refractory chronic sinusitis. As the principles of FESS have evolved over time, so have the instruments uses for sinus surgery. Smaller forceps, mucosal sparing cutting instruments, and powered instrumentation have progressively become available to allow structural preservation.

Balloon catheter dilation (BCD), or Balloon Sinuplasty (BSP), is the newest technology in sinus surgery instrumentation and has been shown to result in the durable dilation of peripheral sinus outflow tracts. Research has shown that sinus surgery with balloon catheter dilation instruments can contribute to a reduction in chronic sinusitis symptoms and improvement in patients' assessed quality of life.

BSP differentiates itself from other sinus instruments by focusing on dilating natural drainage pathways without removing tissue when used as a sole means of treating maxillary, frontal, and sphenoid sinus outflow obstruction.

Recently, BSP has been utilized in the office setting under local anesthesia. Studies have shown that BSP technology can be safely and successfully used in the office setting.

The author has been one of the pioneers of BSP technology since its introduction in 2005. In the last ten years, the author has performed BSP on over a thousand patients and has authored several clinical papers on the use of the technology to manage sinus disease. The session will focus on his journey with BSP from the operating room to In-office. He will be sharing real life patient cases and his learnings on the potential role for BSP in setting up a successful in-office practice. 